\title{
LA PREVENCIÓN Y REPRESIÓN DEL ABUSO \\ DE LA CONTRATACIÓN LABORAL TEMPORAL EN LAS ADMINISTRACIONES PÚBLICAS. COMENTARIO A LA STJUE DE 19 MARZO 2000 (ASUNTOS ACUMULADOS C-103/18 Y C-429/18)
}

\author{
THE PREVENTION AND PUNISHMENT OF ABUSE \\ OF FIXED-TERM EMPLOYMENT CONTRACTS IN THE PUBLIC \\ ADMINISTRATIONS. COMMENT ON THE JUDGEMENT \\ OF THE COURT OF JUSTICE OF THE EUROPEAN UNION \\ OF 19 MARCH 2000 (JOINED CASES C-103/18, C-429/18)
}

\author{
Francisco JaVIER Gómez ABELLEIRA \\ Profesor titular de Derecho del Trabajo \\ Universidad Carlos III de Madrid
}

Recibido: 15.06.2020 / Aceptado: 13.07.2020

DOI: https://doi.org/10.20318/cdt.2020.5649

\begin{abstract}
Resumen: El endémico problema del abuso de la contratación laboral temporal en las administraciones públicas españolas genera un nuevo pronunciamiento del TJUE, que sostiene que la renovación de contratos temporales para cubrir necesidades permanentes contraviene la letra y el espíritu de la Directiva 1999/70. La sentencia analiza la eficacia de algunas medidas legales equivalentes para prevenir los abusos, como en particular la conversión de la relación temporal en "indefinida no fija". Esta medida no permite alcanzar la finalidad perseguida por la cláusula 5 del Acuerdo Marco. Tampoco el consentimiento del trabajador puede considerarse que prive de carácter abusivo al comportamiento del empleador.

Palabras clave: contratos laborales temporales, Directiva 1999/70, administraciones públicas, indefinidos no fijos.

Abstract: The endemic problem of abuse of fixed-term employment contracts in the Spanish public administrations leads to a new judgment of the Court of Justice of the European Union, which holds that the renewal of fixed-term contracts to meet permanent needs is against the letter and purpose of Directive 1999/70. The judgement examines the efficacy of other equivalent legal measures to prevent abuses, such as the transformation of the temporary relationship into a "temporary relationship with a contract of indefinite duration"; this measure is not appropriate for the purpose of attaining the objective pursued by clause 5 of the Framework Agreement. Worker's consent cannot be construed as removing the abusiveness of the employer's behavior.
\end{abstract}

Keywords: fixed-term employment contracts, Directive 1999/70, public administrations, temporary relationship with a contract of indefinite duration.

Sumario: I. Introducción. II La sucesión de contratos temporales. III. Razones objetivas que justifican la contratación temporal. IV. Medidas para prevenir y sancionar los incumplimientos. V. La singular figura del indefinido no fijo. VI. El valor del consentimiento del trabajador. VII. Eficacia de las directivas e interpretación conforme. 


\section{Introducción}

1. La problemática jurídica del trabajo de duración determinada en España ha dado lugar a varias sentencias del Tribunal de Justicia y a un volumen impresionante de litigios en la jurisdicción ordinaria española. Dicha problemática se corresponde con la realidad de un mercado de trabajo dual, en el que trabajadores indefinidos y trabajadores temporales corren suertes diversas, especialmente en tiempos de crisis económica. Esto viene siendo así desde los ya lejanos años 80 del siglo pasado, sin que las múltiples reformas a lo largo de todas estas décadas hayan conseguido resolver el tema ${ }^{1}$.

2. El problema es especialmente delicado en el sector público. Según datos de la última Encuesta de Población Activa, la del primer trimestre de 2020, la tasa de temporalidad del sector privado es del 24,41 por 100 , mientras que la del sector público es superior: 27,49 por 100 . Son datos que sitúan a España a la cabeza de la clasificación de países de la UE por peso del trabajo temporal en su mercado laboral. Nuestra tasa de temporalidad se aleja mucho de la media de la UE o de la Eurozona, que, según la información que proporciona Eurostat, ronda normalmente entre el 11 y el 12 por $100^{2}$. El problema de la temporalidad en el sector público afecta no solo a los trabajadores con relación laboral, sino también a los empleados con relaciones estatutarias o funcionariales sujetas al derecho administrativo.

3. El abuso de la temporalidad por las administraciones públicas españolas es un problema endémico, sobre el que ni el legislador ha acertado (más bien ha abdicado) ni tampoco los tribunales han tenido excesiva fortuna. Baste recordar el episodio rocambolesco del caso Ana de Diego Porras, con una primera controvertida sentencia del TJUE de 14 septiembre 2016 (C-596/14) y una segunda de 21 noviembre 2018 (C-619/17), "casando" lo sentado en la primera ${ }^{3}$, Montero Mateos y Grupo Norte Facility mediante ${ }^{4}$. La historia jurisprudencial de la figura del indefinido no fijo en España, con sus idas y venidas, tampoco es la historia de un éxito y pone de relieve lo trabajoso y desorientador de la creación judicial del derecho 5 .

4. En la reciente sentencia del TJUE de 19 marzo 2020 (asuntos acumulados C-103/18 y C-429/18), se resuelven las cuestiones prejudiciales planteadas por dos Juzgados de lo ContenciosoAdministrativo de Madrid, en sendos supuestos fácticos que implican a personal estatutario temporal del Servicio Público de Salud de la Comunidad de Madrid. Los dos supuestos reflejan una realidad tristemente habitual en el sector público. Como señala uno de los juzgados remitentes,

"el sector público de la salud español se caracteriza, desde hace mucho tiempo, por la desnaturalización de las relaciones de servicio de duración determinada. Los empleados públicos con relaciones de servicio de duración determinada encadenan varias relaciones de servicio, trabajando todos o casi todos los días del año durante varios años, ya que la causa de su nombramiento se cumple siempre. Estos empleados ejercen las mismas funciones que el personal estatutario fijo. De este modo, cubren en realidad necesidades permanentes de personal. Por lo tanto, en esta rama del sector público español existe un problema estructural que se traduce en un elevado porcentaje de empleados públicos temporales, cuya contribución es esencial para el buen funcionamiento de dicho sector, así como en la inexistencia de límites máximos al número de sucesivas relaciones de servicio de duración determinada y en el incumplimiento de la obligación legal de proveer las plazas ocupadas temporalmente por dicho personal mediante el nombramiento de empleados públicos con relaciones de servicio de duración indefinida".

\footnotetext{
${ }^{1}$ Puede verse F.J. Gómez Abelleira, Contratos Laborales y Temporalidad. La politica legislativa de contratación laboral, Tirant lo Blanch, Valencia, 2017.

${ }^{2}$ Temporary employees as percentage of the total number of employees [TESEM110]

3 Vid. A. De la Puebla Pinilla, "Principio y fin de la doctrina «de Diego Porras», o de cómo, en ocasiones, «el sueño de la tutela multinivel produce monstruos»", Revista de Información Laboral, 7/2018.

${ }^{4}$ Sentencias del TJUE ambas de 5 junio 2018 (C-677/16, Montero Mateos) (C-574/16, Grupo Norte Facility).

${ }^{5}$ Vid. A. Desdentado Bonete, "Los indefinidos no fijos: ¿una historia interminable o una historia terminada?", Revista de Información Laboral, 10/2018; I. BeLtrán DE Heredia RuIz, "Indefinidos no fijos: naturaleza jurídica, reconocimiento y extinción”, IUSLabor 3/2017, ISSN 1699-2938, p. 160-190.
} 
5. En el primer asunto, se trata de un informático nombrado personal estatutario temporal interino el 2 de noviembre de 1999, cesado el 28 de diciembre de 2011 y nombrado inmediatamente de nuevo. En todo momento, ocupó un puesto calificado como vacante y desempeñó las mismas funciones. No concurrió al único proceso selectivo organizado para su especialidad entre los años 1999 y 2015 para acceder a la condición de personal estatutario fijo. En diciembre de 2016, el trabajador solicitó a la Comunidad de Madrid que se le reconociera la condición de personal estatutario fijo o, con carácter subsidiario, de empleado público con un estatuto similar, bajo los principios de permanencia e inamovilidad, alegando que era víctima de un comportamiento abusivo por parte de su empleador, derivado de la utilización de sucesivas relaciones laborales de duración determinada. La Comunidad de Madrid denegó su solicitud, al considerar que tal abuso presupone la existencia de sucesivas relaciones laborales de duración determinada y que aquí se trataba de una misma relación de servicio de duración determinada, dado que el segundo nombramiento se produjo debido a una reforma legislativa de las categorías de personal. La Comunidad de Madrid estimó además que el acceso a la condición de personal estatutario fijo está reservado, en principio, a las personas que hayan superado un proceso selectivo y que, conforme al Derecho español, no es posible que un miembro del personal estatutario temporal sea nombrado personal estatutario fijo al margen de un proceso de este tipo. A lo sumo, un miembro del personal estatutario temporal puede ser convertido en «miembro del personal indefinido no fijo» para un puesto hasta que este sea amortizado o adjudicado a un miembro del personal estatutario fijo.

6. El segundo asunto lo protagonizan varias personas que trabajan, desde hace 12 a 17 años, como personal estatutario temporal, ejerciendo funciones de odontólogo y con entre 82 y 227 nombramientos sucesivos, según el caso. Durante los quince años anteriores al auto de remisión solo se convocó un proceso selectivo para la categoría profesional de odontólogo, concretamente en 2015. En julio de 2016, las demandantes solicitaron a la Comunidad de Madrid el reconocimiento de su condición de miembros del personal estatutario fijo o, con carácter subsidiario, de empleados públicos con un estatuto similar, bajo los principios de permanencia e inamovilidad. La Comunidad de Madrid denegó su solicitud.

7. Planteadas las respectivas controversias ante los Juzgados de lo Contencioso-Administrativo de Madrid, estos remiten una larga y prolija serie de cuestiones al TJUE, casi todas en relación con la Directiva 1999/70/CE, por la que se aplica el Acuerdo marco (UNICE, CEEP, CES) sobre el trabajo con contrato de duración determinada (en adelante, ATDD) ${ }^{6}$. La sentencia que se comenta aborda algunos de los más interesantes temas que dicha Directiva plantea: sucesión de contratos temporales, razones objetivas que justifican la contratación temporal, medidas para prevenir y sancionar los incumplimientos, la singular figura del indefinido no fijo, el valor del consentimiento del trabajador. Además, la sentencia aborda una cuestión transversal de interés general, como es la eficacia de las directivas y la obligación judicial de interpretación conforme del derecho nacional.

\section{La sucesión de contratos temporales}

8. Evitar los abusos en la utilización sucesiva de contratos temporales es uno de los dos grandes objetivos de la Directiva 1999/70. La cláusula 1 del ATDD señala que su objeto es mejorar la calidad del trabajo de duración determinada garantizando el respeto al principio de no discriminación y establecer un marco para evitar los abusos derivados de la utilización de sucesivos contratos de duración determinada. Es la cláusula 5 del ATDD la que precisa las diversas técnicas que los Estados miembros pueden utilizar para alcanzar este objetivo: establecer razones objetivas que justifiquen la renovación de los contratos temporales; fijar la duración máxima total de los sucesivos contratos de trabajo de duración determinada; y limitar el número de renovaciones de tales contratos.

\footnotetext{
${ }^{6}$ Para un análisis reciente de la Directiva, J. Pérez Rey, "La contratación de duración determinada (Directiva 1999/70/CE del Consejo, de 28 de junio de 1999)", en M.E. Casas BaAmonde / R. Gil Alburquerque (Dir.), Derecho Social de la Unión Europea. Aplicación por el Tribunal de Justicia, $2^{\text {a }}$ ed., Lefebvre, Madrid, 2019, p. 685-733.
} 
9. La cláusula 5 no determina, sin embargo, cuándo los contratos se entienden sucesivos. Al contrario, confiere a los Estados la facultad para esta determinación. Sobre ello versa la primera de las cuestiones prejudiciales examinadas en la sentencia que se comenta, la cual recuerda que "esta decisión de remitir a las autoridades nacionales la determinación de las modalidades concretas de aplicación del término «sucesivos», a efectos del Acuerdo Marco, se explica por el afán de preservar la diversidad de las normativas nacionales en esta materia". Lo relevante es que, como también recuerda la sentencia, "el margen de apreciación así atribuido a los Estados miembros no es ilimitado, ya que en ningún caso puede llegar hasta el punto de poner en peligro el objetivo o el efecto útil del Acuerdo Marco. En particular, las autoridades nacionales no deben ejercitar esta facultad de apreciación de tal modo que se llegue a una situación que pueda dar lugar a abusos, lo que sería contrario al mencionado objetivo" (apartado 58). Es más, estos límites a la facultad de apreciación conferida a los Estados miembros "se imponen muy especialmente cuando se trata de un concepto clave, como es el de sucesivas relaciones de servicio, que resulta decisivo para determinar el propio ámbito de aplicación de las disposiciones nacionales destinadas a aplicar el Acuerdo Marco" (apartado 60).

10. En el caso que analiza la sentencia, el empleado había prestado los mismos servicios en idéntico puesto durante años, con un nombramiento inicial y un segundo nombramiento años después, pero sin solución alguna de la continuidad. Para el TJ, no considerar que aquí ha existido una sucesión de relaciones temporales respondería a una interpretación demasiado estricta de la noción de relaciones temporales sucesivas, que podría "comprometer el objeto, la finalidad y el efecto útil del mencionado Acuerdo" (apartado 61) y que "permitiría emplear a trabajadores de forma precaria durante años" (apartado 62). La conclusión es, por tanto, clara: estamos ante relaciones laborales sucesivas cuando existen prórrogas o nombramientos sucesivos, por mucho que el empleado ocupe de manera ininterrumpida el mismo puesto y desempeñe las mismas funciones a lo largo de todo el período de empleo.

11. El razonamiento para llegar a esta conclusión se muestra en la sentencia excesivamente escueto y, por momentos, algo tosco, con demasiado apoyo en generalidades. Resulta de más interés en este punto el hilo argumentativo que despliega la Abogada General Sra. Juliane Kokott en sus Conclusiones, presentadas el 17 de octubre de 2019. La cuestión esencialmente controvertida es si el hecho de que la relación temporal se desarrolle de manera formalmente unitaria, sin interrupciones y sin que existan contratos sucesivos, impide considerar la existencia de una "sucesión" de relaciones temporales. La admonición con la que en cierto modo inicia la Abogada General su discurso es significativa: "La protección perseguida por la cláusula 5 del Acuerdo Marco frente a la precarización de la situación de los trabajadores quedaría vacía de contenido si el legislador nacional tuviera la posibilidad de excluir sin más del ámbito de aplicación del Acuerdo Marco ciertas relaciones laborales de larga duración, que formalmente son temporales, declarando, por ejemplo, que, a pesar de sus eventuales modificaciones, constituyen una relación laboral unitaria o configurando desde un inicio estas relaciones laborales, ciertamente temporales, como relaciones laborales por tiempo indefinido hasta la ocupación permanente de la plaza en un momento indeterminado" (apartado 38). La Abogada General aprecia rotundamente este riesgo en el caso que se enjuicia y apunta, sin ambages, al talón de Aquiles del ordenamiento jurídico y de la práctica administrativa españoles: "conforme a las normas nacionales de que se trata, puede suceder que un empleado público que haya sido nombrado como personal estatutario temporal de carácter interino, incluso sin que se renueve formalmente su relación de servicio, siga empleado por tiempo indefinido, si bien no de modo permanente, porque el empleador público, infringiendo la normas legales, no ha celebrado los procesos selectivos necesarios para cubrir las plazas vacantes con carácter permanente" (apartado 39).

12. Sobre esta base, la Abogada General rechaza que al texto del ATDD pueda uno aproximarse de manera puramente formalista: rechaza, en definitiva, un enfoque que "solo contemple la finalización y la subsiguiente creación de una nueva relación jurídica desde un punto de vista formal para admitir que existen diversos contratos o relaciones laborales sucesivos" (apartado 41). En su lugar, lo que procede entender, y esto es especialmente interesante, es que "existen contratos o relaciones laborales sucesivos cuando el régimen de empleo controvertido experimenta una modificación en su contenido, que, en 
contra de la finalidad de la cláusula 5 del Acuerdo Marco, expone al trabajador afectado a una mayor inseguridad"; y esto sucede, siempre según la Abogada General, "cuando la modificación de que se trate tenga por objeto el período de duración del contrato o de la relación laboral, las condiciones para su finalización o las expectativas de lograr un puesto fijo aparejadas al tipo de puesto en cuestión". Pues bien, de todos estos elementos, la Abogada General aprecia que, en el presente asunto, "podría estar en tela de juicio particularmente la expectativa de obtener un puesto fijo" (apartado 42).

13. Elaborando sobre lo anterior, las Conclusiones aportan un argumento sólido para considerar que existe "sucesión" de relaciones temporales, en el sentido de la cláusula 5 del ATDD: dicha sucesión se aprecia cuando la continuación de una relación sin un término claro "se debe a que no se han cumplido las exigencias legales relativas a la cobertura de plazas vacantes. En efecto, el incumplimiento de las mencionadas exigencias legales conduce de hecho a una modificación de la duración de la relación de servicio debido a que esta continúa por tiempo indefinido" (apartado 44). En este contexto, es obvio que la expresión "por tiempo indefinido" debe entenderse como relación "sin un término claro", no como relación fija o permanente.

14. Resulta evidente que solo una interpretación amplia del concepto de sucesión de contratos permite que la Directiva 1999/70 desarrolle todo su potencial normativo y logre su efecto útil, que no es otro que impedir la sustitución de empleo estable por empleo temporal. Aunque la sucesión formal de contratos o nombramientos es el abuso más típico y flagrante, mucho más abusivo resultaría que una misma persona se mantuviera durante años, en una situación formalmente temporal, mediante una única relación o contrato, desarrollando funciones permanentes en una entidad o empresa. No obstante, como se verá más adelante en este comentario, lo esencial es el régimen extintivo del contrato o relación.

\section{Razones objetivas que justifican la contratación temporal}

15. Una de las técnicas o medidas que la cláusula 5 ATDD propone para atajar la utilización abusiva de la sucesión de contratos temporales es el establecimiento de razones objetivas que justifiquen la renovación de tales contratos. Frente a las otras dos técnicas o medidas propuestas por la cláusula 5, esta tiene una naturaleza cualitativa. En efecto, las otras dos técnicas consisten en establecer una duración máxima total de la sucesión de contratos o en establecer un número máximo de renovaciones. Frente al carácter cuantitativo y, por tanto, exacto, claro y predecible de estas dos últimas medidas, la de establecer razones objetivas que justifiquen la renovación presenta un cariz mucho más matizado, pero por ello mismo más intrínsecamente problemático.

16. Es cierto que el TJUE ha dado a este concepto de razones objetivas una interpretación amplia o extensiva, al menos desde la sentencia de 4 julio 2006 dictada en el caso Adeneler (C-212/04). Y es que el concepto no queda exclusivamente referido "a las circunstancias específicas y concretas que caracterizan una determinada actividad y que, por tanto, pueden justificar en ese contexto particular la utilización sucesiva de contratos de trabajo de duración determinada"; sino que se extiende a razones tales como "la persecución de un objetivo legítimo de política social por parte de un Estado miembro" (apartado 66). Sin embargo, esta interpretación extensiva no alcanza a comprender meras disposiciones normativas de los Estados que autoricen "de manera general y abstracta" la sucesión de contratos temporales (apartado 67). La razón es evidente: la exigencia de "razones objetivas" no tiene una naturaleza formal, sino material: se trata de "criterios objetivos y transparentes" que conectan con la idea de "necesidad auténtica" (apartado 68).

17. Desde luego, el derecho español responde a estas exigencias comunitarias. El artículo 15 ET limita la contratación temporal a supuestos que vienen definidos por razones objetivas; ello es así incluso cuando tales razones son susceptibles de interpretaciones más o menos amplias, como las que en relación con temas que no viene al caso precisar más aquí realizan nuestros tribunales nacionales. En el 
caso del empleo público, las circunstancias objetivas son las que amparan el nombramiento de funcionarios interinos (artículo 15 EBEP: RDLeg 5/2015) y las razones de especial confianza las que justifican la figura del funcionario eventual (artículo 12 EBEP). En el caso concreto del personal estatutario de los servicios de salud, el artículo 9 EMPESS (Ley 55/2003) regula las figuras del personal interino, eventual y de sustitución, que responden a razones objetivas, como la existencia de vacante, la cobertura de servicios temporales o extraordinarios o la sustitución de personas de permiso, vacaciones, etc.

18. La sentencia que se comenta no cuestiona de entrada la regulación española en este punto, aunque como luego veremos esto no es del todo así. Tras hacer una revisión de dicha regulación, el TJUE concluye que la norma nacional "no establece una autorización general y abstracta para utilizar sucesivos contratos de trabajo de duración determinada, sino que limita la celebración de tales contratos, en esencia, a los efectos de satisfacer necesidades provisionales" (apartado 71). Lo que sí cuestiona la sentencia es la práctica administrativa consistente en realizar nombramientos de duración determinada, que se renuevan, para desempeñar funciones permanentes y estables de los correspondientes servicios u organismos. La renovación de contratos temporales para cubrir necesidades permanentes y estables contraviene frontalmente la letra y el espíritu de la Directiva 1999/70, en la medida en que esta Directiva y el ATDD establecen el principio de que los contratos de trabajo de duración indefinida "constituyen la forma más común de relación laboral" (apartado 76).

19. Resulta obvio, y está fuera de discusión, que los sucesivos nombramientos de los litigantes en los procedimientos de base "no respondían a meras necesidades provisionales" de la entidad empleadora, "sino que tenían por objeto atender necesidades permanentes y estables de personal del Servicio de Salud" (apartado 78). El dato que a continuación destaca la sentencia es sencillamente sonrojante: "en el momento en que interpusieron sus recursos, todos estos empleados públicos habían prestado servicios para la Comunidad de Madrid durante al menos 12 años consecutivos, [...] algunos de ellos habían sido objeto de más de 200 nombramientos y [...] realizaban tareas propias de la actividad normal del personal estatutario fijo".

20. El problema de esta práctica administrativa atroz -"problema estructural en el sector público de la sanidad española", según cita el TJUE de fuente de los juzgados remitentes- es que, en realidad, demuestra un defecto en el marco normativo, consistente en la "inexistencia de límites máximos al número de relaciones de servicio de duración determinada sucesivas" y en las escasas o nulas consecuencias del "incumplimiento de la obligación legal de proveer los puestos temporalmente cubiertos por dicho personal mediante el nombramiento de empleados públicos con una relación de servicio de duración indefinida" (apartado 79). La conclusión que de todo ello se sigue es la reprobación de un modelo legal y administrativo que no impide, en la práctica, que las administraciones públicas den respuesta mediante sucesivas renovaciones a necesidades permanentes y estables de personal (apartado 80). Como había apuntado la Abogada General en sus Conclusiones, "la observancia de [las] exigencias [normativas] abstractas apenas contribuye a la protección contra la precarización de la situación del interesado cuando la cobertura de la necesidad de personal, en especial por lo que se refiere a la provisión de plazas vacantes de modo permanente, no se garantiza también mediante el cumplimiento efectivo de los requisitos temporales existentes. En caso contrario, la posibilidad, prevista por la normativa nacional, de mantener la relación de servicio hasta que se ocupe con carácter permanente la correspondiente plaza como limitación temporal de carácter meramente formal - supone un cheque en blanco para un número indeterminado de renovaciones o para una continuación indefinida de la relación de servicio temporal, lo que en realidad perpetúa la inseguridad en la que se encuentra el personal temporal" (apartado 54).

21. La cuestión clave es, por tanto, si el sistema nacional ofrece medidas efectivas para prevenir y sancionar los incumplimientos. A esta cuestión dedica la sentencia los extensos apartados 81 a 106. Sin embargo, de entre ellos sobresale un específico tema, que es el de los indefinidos no fijos, que por su importancia conceptual y cuantitativa merece un comentario aparte. 


\section{Medidas para prevenir y sancionar los incumplimientos}

22. Ni la Directiva 1999/70 ni el ATDD que aquella aplica contemplan "sanciones específicas en caso de que se compruebe la existencia de abusos". Sin embargo, es evidente que dejar sin sancionar, o dicho con más precisión, dejar sin consecuencias jurídicas, los abusos que precisamente se pretende combatir sería negar todo efecto útil a la Directiva. Por tanto, y siguiendo doctrina general vertida en multitud de temas, el TJUE apunta que "corresponde a las autoridades nacionales adoptar medidas que no solo deben ser proporcionadas, sino también lo bastante efectivas y disuasorias como para garantizar la plena eficacia de las normas adoptadas en aplicación del Acuerdo Marco" (apartado 86). Entre estos efectos efectivos y disuasorios podría encontrarse, por ejemplo, la conversión de la relación en indefinida, lo cual sería una clara manifestación del principio general de prohibición del fraude: el fraude no puede tener por efecto eludir los que hubieran correspondido de no haberse incurrido en él. Pero esta conversión no viene impuesta por la Directiva, dice el TJUE (apartado 87).

23. Lo que sí se impone es la adopción de medidas adecuadas para prevenir y sancionar los abusos de la temporalidad, pues esto se sigue sin más de la obligación estatal de dar cumplimiento a las Directivas. A este respecto, los tribunales remitentes plantean al TJUE, para su consideración, las siguientes medidas: la organización de procesos selectivos destinados a proveer definitivamente las plazas vacantes ocupadas provisionalmente por empleados temporales, la transformación de las relaciones temporales en relaciones "indefinidas no fijas" y la concesión de una indemnización equivalente a la abonada en caso de despido improcedente. Ante este planteamiento, el TJUE se cuida, en primer lugar, de advertir que "no corresponde al Tribunal de Justicia pronunciarse sobre la interpretación del Derecho interno, ya que esta tarea incumbe a los tribunales nacionales competentes, que deben determinar si lo dispuesto en la normativa nacional aplicable cumple las exigencias [del derecho comunitario]" (apartado 89), y que son los tribunales remitentes los que deben "apreciar en qué medida los requisitos de aplicación y la ejecución efectiva de las disposiciones pertinentes del Derecho interno hacen que estas constituyan una medida apropiada para prevenir y, en su caso, sancionar el uso abusivo de sucesivos contratos o relaciones laborales de duración determinada" (apartado 90). No obstante, el TJUE decide aportar algunas precisiones destinadas a orientar a los tribunales remitentes.

24. Partiendo de la obviedad de que ninguna de las medidas sometidas a la consideración del TJUE está comprendida entre las mencionadas en la cláusula 5, apartado 1, del ATDD, la sentencia se pregunta si tales medidas podrían constituir "medidas legales equivalentes para prevenir los abusos". Respecto de la organización de procesos selectivos que tengan por objeto la provisión definitiva de las plazas ocupadas provisionalmente por empleados temporales, la opinión del TJUE es que "tal medida es adecuada para evitar que se perpetúe la situación de precariedad de dichos empleados, al garantizar que las plazas que ocupan se cubran rápidamente de manera definitiva" (apartado 94). Sin embargo, en el caso concreto se aprecia que, pese a que la normativa aplicable "establece plazos concretos para la organización de tales procesos, en realidad dichos plazos no se respetan y estos procesos son poco frecuentes" (apartado 96). Esta disonancia entre norma y realidad conduce al TJUE a sostener que, en estas circunstancias, la normativa nacional española no resulta adecuada para prevenir la utilización abusiva de las relaciones temporales. Y la clave para sostener esta afirmación es que la previsión normativa no viene respaldada suficientemente por una garantía de que los procesos selectivos se convoquen y organicen efectivamente. Es claro, así, que una normativa como la española no resulta adecuada, pues "su aplicación no tendría ningún efecto negativo para [el] empleador" (apartado 97).

25. Por lo demás, y como bien aprecia la Abogada General en sus Conclusiones (apartados 68-69), el hecho de que se organicen pruebas selectivas para la cobertura definitiva de las plazas no supone una medida de prevención y sanción del abuso. Cuando este ya se ha cometido -y el empleado lleva quizá años en situación de precariedad-, la celebración del proceso selectivo no supone ninguna consecuencia negativa para el empleador, ni tampoco proporciona ninguna forma de reparación singular 
o especial para el trabajador afectado: el hecho de que pueda participar en las pruebas no lo singulariza frente al resto de candidatos que no han sido víctimas de tal abuso (apartado 100).

\section{La singular figura del indefinido no fijo}

26. Un estudio más detenido merece la medida consistente en transformar la relación abusivamente temporal de los empleados públicos en "indefinidos no fijos". La figura del indefinido no fijo, de creación y diseño totalmente jurisprudencial, viene a constituir una de las principales respuestas a la endémica irregularidad en la contratación laboral por parte de las administraciones públicas españolas. A lo largo de ya varias décadas, la figura ha ido perfilándose con importantes vaivenes jurisprudenciales, aunque en el fondo para el Tribunal Supremo, "el contrato indefinido no fijo es precisamente la medida que sanciona el uso abusivo de la contratación temporal en las Administraciones públicas, poniendo fin a la sucesión de contratos temporales mediante la consolidación estable de una situación, que solo terminará con la cobertura o la amortización de la vacante, frente a las que puede reaccionar el trabajador en los términos examinados. Se cumplen así los principios de equivalencia y efectividad, pues la medida se aplica en todas "las situaciones similares" y su única particularidad en las Administraciones públicas tiene la justificación objetiva del respeto a las garantías de acceso al empleo público. Es además una medida efectiva en la medida en que cumple, no una sino todas las exigencias del apartado 5.1 del Acuerdo (razón objetiva, duración máxima y exclusión de las renovaciones)" (STS Soc. 13 mayo 2014, R 2214/2013).

27. Esta mirada tradicional tan benévola, que considera que un contrato temporal es una medida adecuada para prevenir y sancionar el abuso de la temporalidad -pues, en la configuración jurisprudencial, el indefinido no fijo no venía siendo otra cosa que un trabajador temporal- parece desecharse a partir de la STS Soc. 28 marzo 2017 (núm. 257/2017). En ella, se opta por reconocer al trabajador indefinido no fijo cuya relación se extingue por cobertura de la plaza una indemnización extintiva propia de trabajador indefinido (la del despido objetivo procedente), y no la que se le venía anteriormente reconociendo (la de la extinción de contratos temporales por cumplimiento del término, de cuantía menor). El Alto Tribunal hace expreso reconocimiento de que la relación del indefinido no fijo no es una relación temporal.

28. Pese a este avance jurisprudencial interno, al que sí aluden las Conclusiones de la Abogada General (apartados 73-75), el TJUE se limita a tomar nota de lo que señalan los propios juzgados remitentes, que "consideran que esta medida no permite alcanzar la finalidad perseguida por la cláusula 5 del Acuerdo Marco". Primero, porque la transformación "se produce sin perjuicio de la posibilidad de que el empleador amortice la plaza o cese al empleado público con nombramiento de duración determinada de que se trate cuando la plaza se cubra por reingreso del funcionario sustituido". Y segundo, porque, "a diferencia de la transformación, en el sector privado, de los sucesivos contratos de trabajo de duración determinada en contratos de trabajo por tiempo indefinido, la transformación de los empleados públicos con nombramiento de duración determinada en «indefinidos no fijos» no les permite disfrutar de las mismas condiciones de trabajo que el personal estatutario fijo" (apartado 102).

29. En realidad, el debate queda totalmente cercenado de origen por las consideraciones de los tribunales remitentes. El hecho de que no exista igualdad entre fijos e indefinidos no fijos no hubiera debido impedir el debate de fondo acerca de si la aplicación de la indemnización extintiva del despido objetivo (20 días de salario por año de servicio con un máximo de 12 mensualidades) es una medida adecuada para la protección del trabajador, la prevención de abusos y la sanción de la temporalidad injustificada. De todos modos, es probable que este debate fuera rechazado por el TJUE, como prueba el hecho de que, en el asunto que comentamos, desista de entrar en el fondo de la cuestión de si la concesión de una indemnización equivalente a la abonada en caso de despido improcedente (33 días de salario por año de servicio con un máximo de 24 mensualidades) es proporcionada, efectiva y disuasoria como para garantizar la plena eficacia de la cláusula 5 ATDD. Sobre este tema, el TJUE se limita prácticamente a 
señalar que "corresponde a los juzgados remitentes determinar si tal medida es adecuada para prevenir y, en su caso, sancionar tal abuso" (apartado 105).

30. Lo cierto es que, con independencia de las consecuencias en el plano sancionador, parece razonable entender que la aplicación del régimen extintivo del contrato indefinido a una sucesión abusiva de contratos temporales es una medida adecuada y efectiva. Disuasoria por sí sola no tanto, aunque probablemente lo sea si va acompañada del correspondiente aparataje sancionador. Al fin y al cabo, la medida más adecuada para tratar el abuso de la temporalidad es presumir iuris et de iure que el trabajador mantiene con la empresa o entidad empleadora una relación laboral indefinida.

\section{El valor del consentimiento del trabajador}

31. Una de las cuestiones prejudiciales que se plantean en el asunto C-103/18 es si el hecho de que el empleado público haya consentido el establecimiento de diferentes relaciones temporales de servicio priva de carácter abusivo al comportamiento del empleador. No son necesarios conocimientos muy profundos de derecho laboral para anticipar la respuesta más razonable a esta cuestión, máxime si se tiene en cuenta que, como recuerda la sentencia que se comenta, el ámbito de aplicación del ADTT incluye a todos los trabajadores (con algunas excepciones ligadas, sobre todo, a la naturaleza formativa de la relación), "sin establecer diferencias en función del carácter público o privado del empleador para el que trabajan y con independencia de la calificación de su contrato en Derecho interno" (apartado 108).

32. Excluir la aplicación de las reglas anti-abuso del ADTT por el hecho de que el trabajador haya consentido el establecimiento de su relación laboral en los términos propuestos por el empleador sería una solución no solo "manifiestamente contraria a uno de los objetivos del ADTT" (apartado 111), sino simplemente descabellada. El derecho laboral, el nacional y el europeo, se configura como un derecho imperativo y mínimo, que no admite la validez de la renuncia del trabajador a los derechos que, con tal carácter, reconoce el ordenamiento. De este modo, razona el TJUE que el ADTT "se basa implícita pero necesariamente en la premisa de que el trabajador, debido a su posición de debilidad respecto del empleador, puede ser víctima de una utilización abusiva, por parte de este, de relaciones laborales de duración determinada sucesivas, aun cuando el establecimiento y la renovación de esas relaciones laborales se hayan consentido libremente" (apartado 112). Recuerda el TJUE, en la misma línea argumentativa, que "esta situación de debilidad podría disuadir al trabajador de hacer valer expresamente sus derechos frente al empresario, en particular cuando la reivindicación de estos pudiera provocar que quedara expuesto a medidas adoptadas por el empresario que redundasen en perjuicio de las condiciones de trabajo del trabajador" (apartado 113).

33. La conclusión es que aceptar que el consentimiento del trabajador al establecimiento de cualquier condición de empleo o de trabajo inferior a lo mínimos legales imperativos es válido privaría completamente de efecto útil ya no solo al ADTT, sino a todo el derecho laboral.

\section{Eficacia de las directivas e interpretación conforme}

34. La última de las cuestiones de las que se ocupa la sentencia es si un tribunal nacional viene obligado a abstenerse de aplicar una normativa nacional que no es conforme con la cláusula 5 ADTT. La respuesta a esta pregunta pasa por determinar si la citada cláusula es incondicional y suficientemente precisa para que un particular pueda invocarla ante un juez nacional, haciendo valer la cláusula frente al Estado incumplidor que actúa, además, como Estado empleador. Recuérdese que "es jurisprudencia reiterada que, siempre que las disposiciones de una directiva resulten ser, desde el punto de vista de su contenido, incondicionales y suficientemente precisas, los particulares podrán invocarlas frente al Estado, particularmente en su condición de empleador" (STJUE 15 abril 2008, asunto C-268/06, Impact, apartado 57). 
35. La naturaleza no incondicional ni suficientemente precisa de la cláusula 5 , apartado 1 , del ADTT ya había sido establecida en la citada STJUE Impact. En ella el TJUE razonó esa falta de condicionalidad y precisión, argumentando que "no es posible determinar suficientemente la protección mínima que debería aplicarse en cualquier caso con arreglo a la cláusula 5, apartado 1, del Acuerdo marco" (Impact, apartado 78).

36. Con este precedente, la respuesta a esta cuestión es clara: "una disposición del Derecho de la Unión de esta índole, carente de efecto directo, no puede invocarse como tal en un litigio sometido al Derecho de la Unión con el fin de excluir la aplicación de una disposición de Derecho nacional que le sea contraria" (apartado 119) y, por consiguiente, "un tribunal nacional no está obligado a dejar sin aplicación una disposición de su Derecho nacional contraria a la cláusula 5, apartado 1, del Acuerdo Marco" (apartado 120).

37. Lo anterior no es óbice para que el TJUE recuerde a los tribunales remitentes su obligación de interpretar el derecho interno "en la medida de lo posible a la luz de la letra y de la finalidad de la directiva de que se trate para alcanzar el resultado que esta persigue" (apartado 121). Esta obligación de interpretación conforme tiene, como se sabe, sus límites en principios generales como el de seguridad jurídica y no puede servir de base para una interpretación frontalmente contra legem del derecho nacional.

38. En el caso que nos ocupa, sin embargo, no resulta ni contrario a la seguridad jurídica ni contrario a los textos legales españoles una interpretación que establezca la obligación del empleador de abonar al trabajador que ha sido víctima del abuso de la temporalidad una indemnización como si de un trabajador indefinido se tratara. De hecho, la ya citada STS 28 marzo 2017 va ya en esta línea. 